\title{
Quadratic Zeeman effect in Rydberg states of NO
}

\author{
M Raoult ${ }^{1}$, S Guizard ${ }^{2,4}$, D Gauyacq ${ }^{2}$ and A Matzkin ${ }^{3}$ \\ ${ }^{1}$ Laboratoire Aimé Cotton, CNRS, bât. 505, Université Paris XI, 91405 Orsay Cedex, France \\ 2 Laboratoire de Photophysique Moléculaire, CNRS, bât. 210, Université Paris XI, \\ 91405 Orsay Cedex, France \\ ${ }^{3}$ Laboratoire de Spectrométrie physique (CNRS Unité 5588), Université Joseph-Fourier \\ Grenoble-I, BP 87, 38402 Saint-Martin, France
}

Received 25 May 2004

Published 5 January 2005

Online at stacks.iop.org/JPhysB/38/S171

\begin{abstract}
We analyse a resonant-enhanced multiphoton ionization (REMPI) spectrum of the high $n$ (35-50) members of the Rydberg series of NO molecules recorded in a $1 \mathrm{~T}$ external magnetic field. In this range the quadratic Zeeman interaction takes over the Coulomb interaction and gradually scrambles the Coulomb structure of the Rydberg series. A perturbative quantum treatment of the diamagnetic interaction, giving rise to the so-called $l$-mixing and $n$-mixing, shows that the apparent complexity of the higher range of the experimental spectra results essentially from the interplay of two Rydberg series associated with different rotational core states. This perturbative treatment is expected to gradually break down and a semiclassical approach is proposed to understand the underlying physical pattern governing this higher spectral range.
\end{abstract}

\section{Introduction}

In the last two decades, the study of highly excited Rydberg atoms in a strong magnetic field has been one of the most challenging problems in atomic physics. The usual spectral regularity of Rydberg series $n \ell$ ( $n$ principal quantum number, $\ell$ angular momentum of the Rydberg electron), associated with the Coulomb field, is gradually destroyed by the quadratic Zeeman interaction. The complexity and the strong dependence with the magnetic field of the corresponding observed spectra made Rydberg systems good candidates to search for evidence or a signature of quantum chaos, in a system which is already known to be classically chaotic. Another important advantage of choosing Rydberg states is that one can observe the evolution of the competition between the Coulomb and the magnetic fields by exploring the Rydberg series from the low $n$ (principal quantum number) to the high $n$ values. On one hand the low $n$ regime is governed by the Coulomb interaction and the magnetic interaction is treated as a perturbation. This is the regime for which Paschen-Back or linear Zeeman effects (LZE) are observed. Then an intermediate regime is reached when the quadratic Zeeman

4 Present address: Laboratoire des Solides irradiés, CEA/CNRS/Ecole Polytechnique, 91128 Palaiseau, France 
effect (QZE) interaction becomes of the same order as the Rydberg energy splitting between two successive members of the series. Finally, the high $n$ regime occurs when the Coulomb interaction becomes a perturbation and the structure of the spectra is imposed by the magnetic field. In this case the relevant picture is given by considering Rydberg series associated with the different Landau levels and coupled by Coulomb interaction. This last situation was extensively studied in the nineties by different groups [1-5].

In this paper, we analyse the experimental spectrum of NO Rydberg states in a $1 \mathrm{~T}$ field and we propose a perturbative treatment well suited for an intermediate field regime. As compared to Rydberg atoms in a magnetic field, very few theoretical or experimental studies have been carried out on molecules. Monteiro and Taylor [6] first proposed a theoretical treatment of the QZE in the $\mathrm{H}_{2}$ molecule in the strong field regime (up to $5 \mathrm{~T}$ ). More recently, an experimental approach to magnetic effects on a Rydberg molecule was performed by Takazawa and Abe $[7,8]$. These authors studied NO under various magnetic fields, scanned Rydberg states below the ionization potential (IP) and observed Landau structures above IP. They used a two-colour excitation scheme similar to the one employed in this work, and their spectra recorded below IP encompassed the same energy range as the one presented in our work. Nevertheless, because of the poor resolution of their spectra, they did not attempt to interpret the Zeeman structure of the high Rydberg levels below IP. Indeed the complexity of the diamagnetic structure in such Rydberg molecules requires a very high resolution in order to understand the interplay between the Coulomb interaction, the molecular field anisotropy and the magnetic perturbation.

More precisely, as in atoms, the QZE interaction first induces a mixing between all the allowed $n \ell$ sublevels of the same parity associated with the same $n$ value. With increasing $n$ this interaction becomes of the same order of magnitude as the energy separation between two successive members of the Rydberg series and gives rise to the so-called $n$-mixing regime. In other words, the usual set $n \ell m_{\ell}$ of quantum numbers characterizing an atomic Rydberg level becomes less and less relevant as it approaches IP.

The study of QZE in a molecule is more difficult than in an atom because a third interaction competes with the two previous ones, namely the interaction of the Rydberg electron with the rotation of the nuclei. The interaction between the Rydberg electron and the molecular core may be understood as a molecular field acting as a torque on the Rydberg electron and vice versa. The strength of the torque depends on the anisotropy of the molecular field, which is reflected in the lift of degeneracy of the Rydberg electron energy according to its projection $\lambda=\sigma, \pi$ upon the internuclear axis. A molecular Rydberg level $N^{+} n \ell m_{\ell}$ is characterized by a fourth additional quantum number, namely the angular momentum $N^{+}$describing the rotational state of the ionic core. Since the pioneering work of Fano [9], it is well established that the anisotropy of the molecular field already mixes the different $N^{+}$channels. The study of the linear Zeeman effect in $\mathrm{H}_{2}$ [10] or $\mathrm{NO}$ [11] molecules has shown that this rotational coupling mixes the different projections $m_{\ell}$ of the angular momentum $\ell$ of the Rydberg electron quite unlike the atomic case. Note however that this complication only arises at low $\ell$ values $(\ell \leqslant 3)$. Indeed, for the high $\ell$ values, the centrifugal barrier is screening all the molecular core effects and they behave just like atomic hydrogenic Rydberg states.

Nevertheless, low $\ell$ Rydberg states in molecules play an important role since they are the ones which carry significant oscillator strength in the observed Rydberg series spectra from the intermediate low $n$ Rydberg state used in two-colour REMPI experiments. As in the atomic case (see O'Mahony and Taylor [12]), QZE further dilutes the oscillator strength of these low $\ell$ states into all the allowed higher $\ell$ states. These interactions induce a distortion of the Coulomb pattern and even its disappearance. Our approach closely follows Lu and Rau's [13] multichannel quantum defect theory (MQDT) atomic treatment. The QZE matrix 
elements are evaluated in the basis set $\mid N^{+} \ell m_{\ell}>$ introduced in our former MQDT treatment of molecular LZE [11] which constitutes a good zero-order determination of all the $N^{+} n \ell m_{\ell}$ Rydberg levels lying in the energy range scanned in the present experiment.

We are aware that this perturbative treatment will gradually break down at high Rydberg electron energies especially when the Coulomb pattern completely disappears. In the studies of atoms in a strong external field, semiclassical treatments have been extensively used to bring physical insight into the modulations of the intensity observed in photoabsorption spectra, the most famous being the Landau oscillations observed above the ionization threshold. Matzkin et al $[14,15]$ have used and extended such semiclassical methods to account for molecular effects. In their study of QZE in the scaled $\mathrm{H}_{2}$ molecule, they have shown that their method is in excellent agreement with quantal calculations. This is why, in addition to the perturbative approach proposed below, a semiclassical treatment is also described in this paper in order to deal with the high density level energy range, and to give an alternative interpretation of the dynamics in terms of closed classical orbits.

\section{Experimental setup}

The spectral congestion in the energy region of interest for molecular Rydberg states is now currently reduced by using highly selective excitation as the resonant two-colour multiphoton ionization technique. The experimental setup used in this study has been described elsewhere [16]. In brief, the experiment was carried out in a 'magnetic bottle' time of flight electron spectrometer. A highly inhomogeneous magnetic field diverging from the nominal value of $1 \mathrm{~T}$ in the ionization region down to $10^{-3} \mathrm{~T}$ in the drift tube was used to parallelize the electron trajectories without changing their initial velocity. However, for this study, the magnetic field could be considered as constant and equal to $0.93 \mathrm{~T}$ in the effective excitation volume. The pump and the probe laser intensities were kept as low as possible in order to avoid saturation and broadening effects in the double resonance spectra: typical pulse energies were 5-15 $\mu \mathrm{J}$ for the pump laser and 50-100 nJ for the probe laser. The two excimer pumped (Lambda Physics EMG 101) dye lasers were operated with a laser bandwidth of $0.08 \mathrm{~cm}^{-1}$ for the pump laser (Quantel TDL III) and $0.04 \mathrm{~cm}^{-1}$ for the probe laser (Lambda Physic FL 2002, including an intra-cavity etalon), respectively. For some probe transitions, a frequency doubling crystal was used.

The excitation process involves two steps: in the first step, an intermediate rotational Zeeman sublevel of the $\mathrm{A}^{2} \Sigma^{+}(3 \mathrm{~s} \sigma), v=1$ state of NO is pumped via a two-photon transition with the pump laser. In the second step, the high lying $n \mathrm{p}$ and $n \mathrm{f}$ Rydberg states with $v^{\prime}=1$ are excited via a one-photon transition with the probe laser. These excited states are further ionized via vibrational autoionization into the $v^{+}=0$ ionization continuum (the ionization mechanism was characterized by photoelectron spectroscopy [16]). In this experiment, the two-photon pump step is mainly responsible for the high selectivity of the process, since only one specific $N, M_{S}$ rotational-Zeeman component of the A state was populated. In each $M_{S}= \pm 1 / 2$ component, the $M_{N}$ sublevels were not resolved but their population was taken into account in the spectral simulation described below. The last step, i.e. the autoionization step of the high Rydberg states, was saturated and therefore did not strongly affect the intensity nor the bandwidth of the peaks in the observed spectra. Consequently, the double resonance spectra which will be presented below can be considered as only governed by the RydbergRydberg transition from the A state (probe state) in a nominal magnetic field of $1 \mathrm{~T}$. Although special care was taken in order to keep the Rydberg-Rydberg transition unsaturated, saturation effects on the strongest lines cannot be ruled out. 

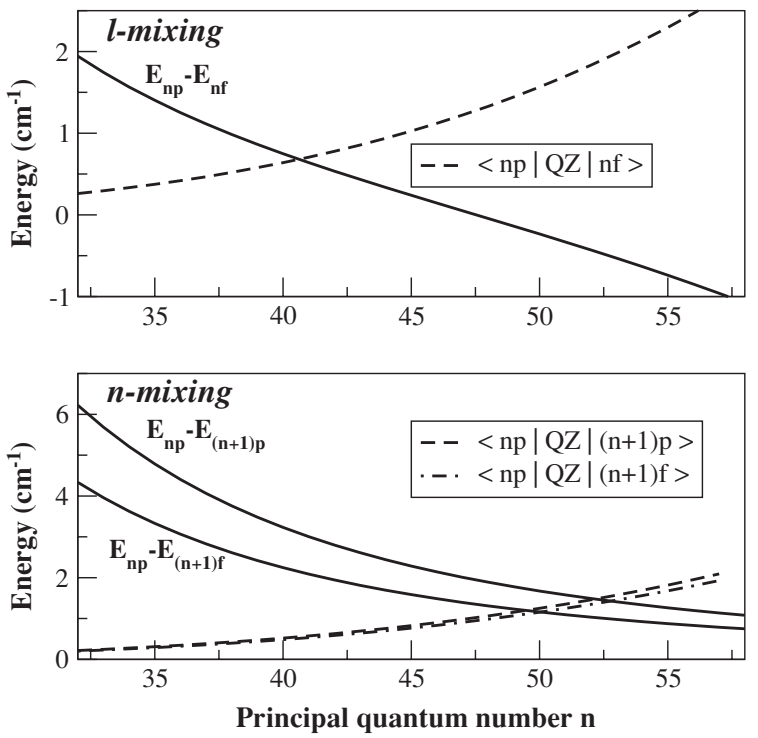

Figure 1. Top panel, $l$-mixing: QZ interaction between $n \mathrm{p}$ and $n \mathrm{f}$ levels as a function of the principal quantum number $n$ in a 1 T magnetic field. This interaction is compared with the energy separation $E_{n \mathrm{p}}-E_{n \mathrm{f}}$ to show from which $n$ values the $l$-mixing, within a $n$ manifold, becomes effective. $E_{n \ell}$ energies account for the diagonal QZ shift. Bottom panel, $n$-mixing: the QZ interactions between two successive $n$ manifolds, indicating the $n$ values from which $n$-mixing is expected.

\section{Perturbative treatment}

\subsection{Basis wavefunctions and dipole moments}

Let us first recall the magnetic perturbation operator for a one-electron system, namely

$$
W_{\mathrm{mag}}=\mu_{B}\left(L_{Z}+2 S_{Z}\right) \cdot B+\frac{e^{2}}{8 m} B^{2} r^{2} \sin ^{2} \theta
$$

where $\mu_{B}$ is the Bohr magneton, $B$ is the field strength, $e$ and $m$ are the charge and the mass of the electron, respectively, and $r$ and $\theta$ are the polar coordinates of the electron. The $Z$-axis of the laboratory frame is chosen along the field axis. $L_{Z}$ and $S_{Z}$ are respectively the electronic and spin angular momenta along the $Z$-axis. The first term of equation (1) is responsible for the LZE. In a $1 \mathrm{~T}$ field this perturbation is roughly of the order of $\mu_{B} B \sim 0.5 \mathrm{~cm}^{-1}$. The second term in equation (1) represents the QZE. It is roughly proportional to $n^{4} B^{2}$. As shown in figure 1 , in a $1 \mathrm{~T}$ field, this perturbation is negligible for low $n$ states, becomes comparable to the LZ perturbation for $n \geqslant 35$ and finally is responsible for the dominant perturbation of the molecular system for high $n$.

Our approach is quite equivalent to that of Lu and Rau in the treatment of Rydberg atoms in external fields [13]. In a previous work the LZE has been taken into account in a MQDT approach leading to Rydberg series converging to the field dressed ionic channel $\left|N^{+} m_{\ell}\right\rangle$ $[10,11]$. The resulting $n \ell$ Rydberg wavefunction $\Psi^{n \ell, M_{N}}$ associated with the energy $E_{n \ell}$ is given by

$$
\Psi^{n \ell, M_{N}}(r, \theta, \phi)=\sum_{N^{+} m_{\ell}} Z_{N^{+} m_{\ell}}^{n \ell, M_{N}} R_{v_{N^{+} m_{\ell}}^{n \ell}}(r) Y_{\ell m_{\ell}}(\theta, \phi)\left|N^{+} m_{\ell}\right\rangle^{M_{N}}
$$


where the $Z_{N^{+} m_{\ell}}^{n \ell, M_{N}}$ coefficients represent the contribution of each dressed channel to the wavefunction [10,11]. This wavefunction will be used as the zero-order basis function for the perturbative treatment of the QZE. As noted above, the QZE interaction given in equation (1) is treated in this work as a perturbation on the LZE levels. Figure 1 shows the limitation of this approach with increasing $n$ values. $M_{N}$ is the projection of the total angular momentum $N$ of the molecule along the $B$ field. The different $N$ values are mixed by the field, but the projection quantum number $M_{N}$ remains a good quantum number. In each channel, the electron radial wavefunction is a Coulomb function $R_{v_{N^{+} m_{\ell}}^{n \ell}}(r)$, the angular wavefunction is taken as the spherical harmonic $Y_{\ell m_{\ell}}(\theta, \phi) .\left|N^{+} m_{\ell}\right\rangle^{M_{N}}$ represents the dressed ionic core wavefunction indexed by its rotational quantum number $N^{+}$and $m_{\ell}$ which indicates that the ionization limit is shifted by $m_{\ell} \mu_{B} B$. Finally $v_{N^{+} m_{\ell}}^{n \ell}$ is the effective principal quantum number of the $n \ell$ Rydberg level of energy $E_{n \ell}$ associated with the $\left|N^{+} m_{\ell}\right\rangle^{M_{N}}$ ionization threshold. Two cases must be considered. On one hand, the penetrating $\ell \leqslant 3$ and low $n$ Rydberg states for which the molecular anisotropy mixes $N^{+}$and $m_{\ell}$. On the other hand, the non-penetrating high $\ell$ Rydberg levels $(\ell \geqslant 5)$ for which the centrifugal barrier is so important that the Rydberg electron no longer 'feels' the anisotropy of the molecular field. As a consequence, these high $\ell$ Rydberg levels converging towards the $N^{+} \ell m_{\ell}$ dressed threshold may be described as pure hydrogenic Rydberg states. In this case, all the quantum defects associated with this high $\ell$ value are taken to be zero. The expansion of equation (2) is then reduced to a single term

$$
\Psi^{n \ell, M_{N}}(r, \theta, \phi)=R_{n \ell}(r) Y_{\ell m_{\ell}}(\theta, \phi)\left|N^{+} m_{\ell}\right\rangle^{M_{N}} .
$$

Within this basis set it was shown in a previous paper that the two strongly allowed transitions occur from the intermediate $\mathrm{A}^{2} \Sigma^{+}(3 \mathrm{~s} \sigma, N=3)$ level towards the $N^{+}=3, n \mathrm{p}$ series and the $N^{+}=5, n \mathrm{f}$ series [17]. These series are shown in addition to the weaker $N^{+}=1, n \mathrm{p}$ series in figure 2 . The LZE intensity pattern, shown in this figure, has been determined previously in the MQDT framework [11]. In the previous work, $p$ and $f$ Rydberg series were studied separately and the electronic radial factor for the dipole moment was omitted. In this work, $\mathrm{p}$ and $\mathrm{f}$ series are coupled by the QZ interaction and thus the relative magnitude of $\mathrm{p}$ and $\mathrm{f}$ radial components of the dipole moment must be accounted for. Observation of $\mathrm{f}$ series from the $\mathrm{A}^{2} \Sigma^{+}$state is due to a small known d contribution [18] which has been determined ab initio by Kaufman et al [19]:

$$
\left.\left.\left.\right|^{2} \Sigma^{+}\right\rangle=\cos \alpha|\mathrm{s} \sigma>+\sin \alpha| \mathrm{d} \sigma\right\rangle
$$

In this work, the mixing angle $\alpha=15^{\circ}$ leading to relative weights of $94 \%$ for s and $6 \%$ for $\mathrm{d}$ components has been adjusted to the experimental $\mathrm{p}: \mathrm{f}$ levels intensity ratio observed in non-overlapping spectral regions, in good agreement with the theoretical value $(5 \% \mathrm{~d}$ in the A state) [19]. Accounting for the weak molecular $\ell$-mixing in the initial A state, the dipole moment associated with the $N^{+} m_{\ell}$ component can be written as

$$
\begin{aligned}
D_{N^{+} m_{\ell}}^{\mathrm{p}, M_{N}} & =\cos \alpha\langle\mathrm{p}|r| \mathrm{s}\rangle d_{N^{+} m_{\ell}}^{\mathrm{p}, M_{N}} \\
D_{N^{+} m_{\ell}}^{\mathrm{f}, M_{N}} & =\sin \alpha\langle\mathrm{f}|r| \mathrm{d}\rangle d_{N^{+} m_{\ell}}^{\mathrm{f}, M_{N}}
\end{aligned}
$$

where the dipole moments $d_{N^{+} m_{\ell}}^{\ell}$ are given by equation (12) of [11]. The radial parts of the dipole transition $\langle\mathrm{p}|r| \mathrm{s}\rangle$ or $\langle\mathrm{f}|r| \mathrm{d}\rangle$ can be evaluated using the hydrogenic approximation of the radial wavefunctions [20] in the initial and final states. This estimation gives $\langle\mathrm{f}|r| \mathrm{d}\rangle=2\langle\mathrm{p}|r| \mathrm{s}\rangle$ assuming that the quantum defects for the $3 \mathrm{~s} \sigma$ and the $3 \mathrm{~d} \sigma$ contributions are 1 and 0 respectively. The transition moment from the $\mathrm{A}^{2} \Sigma$ state to the $n \ell$ Rydberg levels is thus

$$
D_{n \ell}^{M_{N}}=\sum_{N^{+} m_{\ell}} Z_{N^{+} m_{\ell}}^{n \ell, M_{N}} D_{N^{+} m_{\ell}}^{\ell, M_{N}}
$$




\section{$\mathrm{N}^{+}=1$ \\ /////}

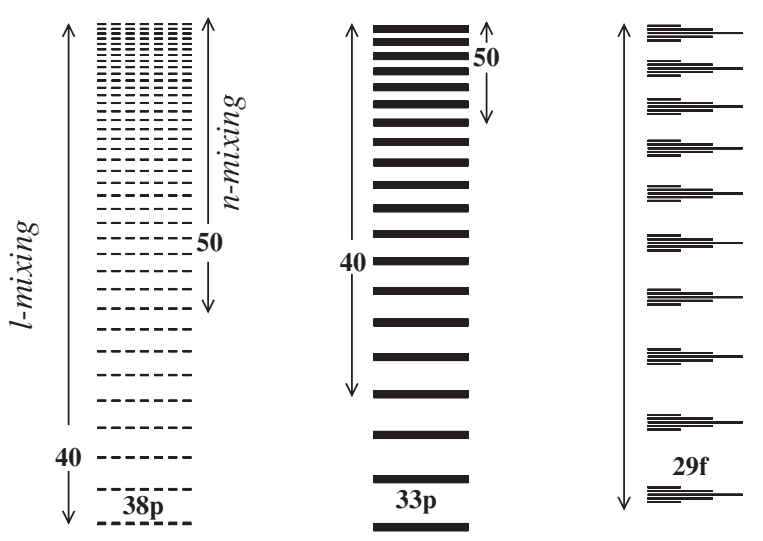

Figure 2. LZE intensity pattern expected from excitation via $\mathrm{A}^{2} \Sigma^{+}$using a linearly polarized light. Only the most intense Rydberg series are reported. The thickness of the line sketching a Rydberg level is proportional to the LZE intensity. For each rotational channel we have reported on the left (resp. on the right) an arrow indicating the range in which $l$-mixing (resp. $n$-mixing) effects are expected to take place in each series.

Note however that the high $\ell$ value Rydberg series $(\ell \geqslant 5)$ are not optically accessible from the $\mathrm{A}^{2} \Sigma, N=3$, in other words $D_{n \ell}^{M_{N}}=0$ for $\ell \geqslant 5$.

\subsection{QZE interaction matrix elements}

As implemented by Lu and Rau [13] (see their equations (11) and (12)), the QZE interaction matrix elements are built up from the LZE energies and the eigenvectors $n N^{+} \ell m_{\ell}$ determined using the LZE-MQDT treatment $[10,11]$ and take the form

$$
\begin{aligned}
\left\langle n \ell\left|r^{2} \sin ^{2} \theta\right| n^{\prime} \ell^{\prime}\right\rangle & =\sum_{N^{+} m_{\ell}} \sum_{N^{+} m_{\ell^{\prime}}} Z_{N^{+} m_{\ell}}^{n \ell} Z_{N^{+} m_{\ell^{\prime}}}^{n^{\prime} \ell^{\prime}} \\
\times & \left.\times R_{v_{N^{+} m_{\ell}}^{n} \ell}\left|r^{2}\right| R_{v_{N^{+} m_{\ell^{\prime}}}^{n^{\prime} \ell^{\prime}}}\right\rangle\left\langle\ell m_{\ell}\left|\sin ^{2} \theta\right| \ell^{\prime} m_{\ell^{\prime}}\right\rangle\left\langle N^{+} \mid N^{+^{\prime}}\right\rangle .
\end{aligned}
$$

The last bracket demonstrates that the QZ interaction is diagonal within each rotational channel $N^{+}$. Analysis of the angular $\left\langle\ell m_{\ell}\left|\sin ^{2} \theta\right| \ell^{\prime} m_{\ell^{\prime}}\right\rangle$ matrix element shows that this matrix element 
connects only levels with $\Delta \ell=\ell-\ell^{\prime}=0, \pm 2$ and $\Delta m_{\ell}=0 \operatorname{since} \sin ^{2} \theta \sim Y_{2,0}(\theta, \phi)$. These selection rules simplify the basic diamagnetic matrix element as follows:

$\left\langle n \ell\left|r^{2} \sin ^{2} \theta\right| n^{\prime} \ell^{\prime}\right\rangle=\sum_{N^{+} m_{\ell}} Z_{N^{+} m_{\ell}}^{n \ell, M_{N}} Z_{N^{+} m_{\ell}}^{n^{\prime} \ell^{\prime}, M_{N}}\left\langle R_{\nu_{N^{+} m_{\ell}}^{n \ell}}\left|r^{2}\right| R_{v_{N^{+} m_{\ell}}^{n^{\prime} \ell^{\prime}}}\right\rangle\left\langle\ell m_{\ell}\left|\sin ^{2} \theta\right| \ell^{\prime} m_{\ell}\right\rangle$.

As already stressed by Lu and Rau [13] this equation clearly shows that the diamagnetic interaction is a direct measure of the amount of inter-channel coupling, originating only from the anisotropy of the molecular field, through the $Z$ mixing coefficients. In addition, due to the magnitude of the radial matrix element $\left\langle R_{v_{N^{+} m_{\ell}}^{n \ell}}\left|r^{2}\right| R_{v_{N^{\prime} m_{\ell}}^{n^{\prime} \ell^{\prime}}}\right\rangle$, the coupling is dominant between the states of the same $n$.

So let us consider the diagonal matrix elements of equation (8) given by

$$
\left\langle n \ell\left|r^{2} \sin ^{2} \theta\right| n \ell\right\rangle=\sum_{N^{+} m_{\ell}}\left(Z_{N^{+} m_{\ell}}^{n \ell, M_{N}}\right)^{2}\left\langle R_{\nu_{N^{+} m_{\ell}}^{n \ell} \ell}\left|r^{2}\right| R_{\nu_{N^{+} m_{\ell}}^{n \ell}}\right\rangle\left\langle\ell m_{\ell}\left|\sin ^{2} \theta\right| \ell m_{\ell}\right\rangle .
$$

In the high $\ell$ level case, the sum is reduced to one hydrogenic term, $R_{v_{N^{+} m \ell}^{n \ell}}=R_{n \ell}$, the corresponding QZ matrix elements may be determined analytically, as reported, for example, in the Garstand review paper [21].

$$
\begin{aligned}
& \left\langle R_{n \ell}\left|r^{2}\right| R_{n \ell}\right\rangle=\frac{n^{2}}{2}\left(5 n^{2}+1-3 \ell(\ell+1)\right) \\
& \left\langle\ell m_{\ell}\left|\sin ^{2} \theta\right| \ell m_{\ell}\right\rangle=2 \frac{m_{\ell}^{2}+\ell^{2}+\ell-1}{(2 \ell+3)(2 \ell-1)} .
\end{aligned}
$$

In the $\ell=1$ and $\ell=3$ cases the diagonal diamagnetic matrix element equation (9) is evaluated within the LZE calculations. The difficulty stands in the evaluation of the radial integrals $\left\langle R_{v_{N^{+} m_{\ell}}^{n \ell}}\left|r^{2}\right| R_{v_{N^{+} m_{\ell}}^{n \ell}}\right\rangle$ when the effective quantum number $v_{N^{+} m_{\ell}}^{n \ell}$ is not an integer. It is often suggested to use $v_{N^{+} m_{\ell}}^{n \ell}$ instead of $n$ in the hydrogenic formulae $[13,21]$. Here we rather use a two-dimensional spline interpolation procedure in order to evaluate these radial integrals.

A rapid inspection of equation (8) shows that in principle a $n \ell$ level may interact with any $n^{\prime} \ell^{\prime}$ level. This would lead to a huge diamagnetic interaction matrix which would be difficult to diagonalize. However, there is an important hierarchy in the magnitude of the offdiagonal coupling matrix elements. Let us again begin with the high $\ell$ Rydberg state for which equation (8) is reduced to one term, namely

$$
\left\langle n \ell\left|r^{2} \sin ^{2} \theta\right| n^{\prime} \ell^{\prime}\right\rangle=\delta_{N^{+} N^{+}} \delta_{m_{\ell} m_{\ell^{\prime}}}\left\langle R_{n \ell}\left|r^{2}\right| R_{n^{\prime} \ell^{\prime}}\right\rangle\left\langle\ell m_{\ell}\left|\sin ^{2} \theta\right| \ell^{\prime} m_{\ell}\right\rangle .
$$

As a result the molecular high $\ell$ Rydberg states may be treated as three independent atomic-like Rydberg series, each of them belonging to one of the three rotational channels, as depicted in figure 2. All these high $\ell$ states are degenerate in energy (without the diagonal QZ contribution) and the primary manifestation of the diamagnetic interaction is the so-called $\ell$-mixing, arising from the QZ off-diagonal matrix elements between levels whose $\ell$ values differ by $\Delta \ell= \pm 2$ within the same $n$ manifold. Those matrix elements are given by [21]

$$
\begin{aligned}
& \left\langle R_{n(\ell-2)}\left|r^{2}\right| R_{n \ell}\right\rangle=\frac{5 n^{2}}{2}\left[\left(n^{2}-\ell^{2}\right)\left(n^{2}-(\ell-1)^{2}\right)\right]^{1 / 2} \\
& \left\langle(\ell-2) m_{\ell}\left|\sin ^{2} \theta\right| \ell m_{\ell}\right\rangle=-\left[\frac{\left(\ell^{2}-m_{\ell}^{2}\right)\left((\ell-1)^{2}-m_{\ell}^{2}\right)}{(2 \ell-3)(2 \ell-1)^{2}(2 \ell+1)}\right]^{1 / 2} .
\end{aligned}
$$



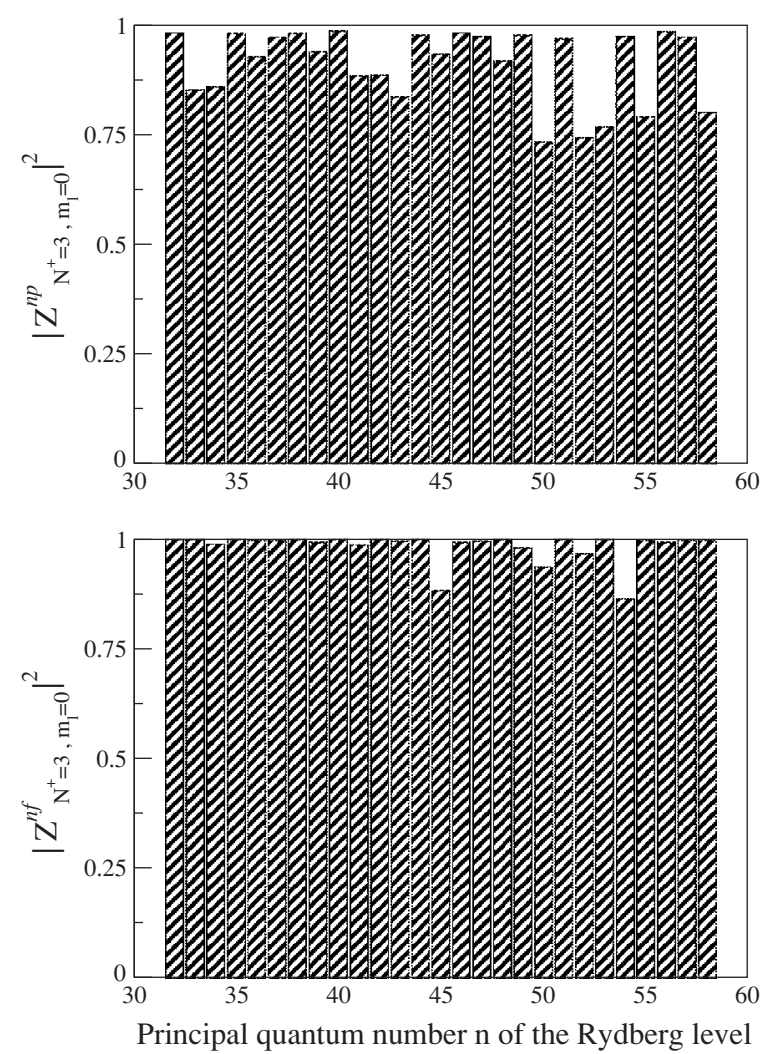

Figure 3. Weight of the major $\left|N^{+}=3, m_{\ell}=0\right\rangle$ contribution to the LZE-MQDT wavefunction equation (2) which allows us to assign the $\left|n \mathrm{p}, N^{+}=3, m_{\ell}=0\right\rangle$ (resp. $\left|n \mathrm{f}, N^{+}=3, m_{\ell}=0\right\rangle$ Rydberg series in the top (resp. bottom) panel.

The next important coupling in strength is the so-called $n$-mixing, i.e. when $n$ differs from $n^{\prime}$ in the radial integral $\left\langle n \ell\left|r^{2}\right| n^{\prime} \ell^{\prime}\right\rangle$ of equation (12). This integral is no longer analytical and is again evaluated in the Oertel and Shomo Coulomb approximation [20]. This approximation indicates that only $\left\langle n \ell\left|r^{2}\right| n \pm 1 \ell \pm 2\right\rangle$ integrals are important. Indeed the radial integrals corresponding to $\Delta n= \pm 2$ integrals are weaker than those corresponding to $\Delta n= \pm 1$, by a factor of around 5. Consequently, off-diagonal matrix elements have been considered only between $(n, \ell)$ and $(n \pm 1, \ell \pm 2)$ Rydberg levels converging to a given $\left(N^{+} m_{\ell}\right)$ dressed ionization limit.

In the low $\ell$ case, the evaluation of the non-diagonal diamagnetic interactions from equation (8) is a priori more difficult. The anisotropy of the molecular field mixes different channels and several non-zero contributions to the LZE-MQDT wavefunction (equation (2)) may be expected. However in the NO case this anisotropy is rather weak and the multichannel wavefunction (equation (2)) has really only one very dominant contribution. This can be checked quantitatively in figure 3 , which shows the weight $\left(Z_{N^{+} m_{\ell}}^{n \ell}\right)^{2}$ of the dominant contribution to the LZ-MQDT wavefunction. More specifically, in the upper panel of figure 3 the weight of the $\left|N^{+}=3, m_{\ell}=0\right\rangle$ contribution allows us to assign this level as belonging to the $n \mathrm{p}$ Rydberg series converging to the $\left|N^{+}=3, m_{\ell}=0\right\rangle$ dressed ionization limit, which dominates the spectrum intensity. In the lower panel of figure 3 the weight of this same contribution in the $n \mathrm{f}$ Rydberg series is reported. Figure 3 clearly demonstrates that one 
contribution dominates for nearly all the $n \mathrm{f}$ levels and the majority of the $n \mathrm{p}$. Thus the situation for low $\ell$ values is indeed not very different from that of the high $\ell$ values case, at least for the NO molecule. In other words a $n \mathrm{p}$ (resp. $n \mathrm{f}$ ) level is only considered to be coupled by the diamagnetic term to $(n \pm 1)$ p (resp. $(n \pm 1) f)$ or $(n \pm 1)$ f (resp. $(n \pm 1)$ p) level within the same $\left(N^{+} m_{\ell}\right)$ dressed ionization channel. The fact that the effective quantum number $v_{N^{+} m_{\ell}}^{n \ell}$ is not an integer is accounted for by the same two-dimensional spline procedure as for the evaluation of diagonal QZ matrix elements.

In summary the structure of the QZ interaction matrix is built up with the LZE basis set within each $N^{+} m_{\ell}$ manifold for a given $M_{N}$, the only good quantum number. Consequently, the QZE in the NO molecule is treated within three distinct atomic-like series for each $\mathrm{N}^{+}$ threshold, as shown in figure 2 (and this is repeated for each $m_{\ell}$ value). Thus our treatment pre-requires that every level in the spectral range of interest must be assigned to a dressed ionization limit. This assignment is performed by looking for the largest contribution $Z_{N^{+} m_{\ell}}^{n \ell}$ of the LZ wavefunction (equation (2)) and the $n \ell$ solution is accordingly labelled $n \ell N^{+} m_{\ell}$, with the energy $E_{n \ell}^{N^{+} m_{\ell}}$ and associated dipole transition moment $D_{n \ell}^{N^{+} m_{\ell}, M_{N}}$ (equation (6)). Finally from the $n$-dependence of the diamagnetic matrix elements, a different QZ regime is expected within each rotational channel: $\ell$-mixing and $n$-mixing regimes appear in different regions of $n$, respectively, as portrayed in figure 2 .

\subsection{Perturbative spectra}

Within all the approximations discussed in the previous section, the diagonalization of the QZ matrix leads to the perturbed level energies $E_{\alpha}^{N^{+} m_{\ell}, M_{N}}$ and the eigenvectors $U_{n \ell, \alpha}^{N^{+} m_{\ell}, M_{N}}$, where $\alpha$ is the eigenvalue index. Correspondingly, the oscillator strength associated with each perturbed level is modified from the zero-order LZE oscillator strength as follows:

$$
\begin{aligned}
& f_{\alpha}^{N^{+} m_{\ell}, M_{N}}=\frac{2}{3} E_{\alpha}^{N^{+} m_{\ell}, M_{N}}\left(D_{\alpha}^{N^{+} m_{\ell}, M_{N}}\right)^{2} \\
& D_{\alpha}^{N^{+} m_{\ell}, M_{N}}=\sum_{n \ell} U_{n \ell, \alpha}^{N^{+} m_{\ell}, M_{N}} D_{n \ell}^{N^{+} m_{\ell}, M_{N}},
\end{aligned}
$$

where $D_{n \ell}^{N^{+} m_{\ell}, M_{N}}$ is the zero-order LZE transition dipole moment [10, 11]. As sketched in figure 2 , only the $\ell=1$ and $\ell=3$ Rydberg series are optically accessible from the $\mathrm{A}^{2} \Sigma$ initial state. Thus the diamagnetic interaction dilutes the intensity pattern shown in this figure into the higher $l$ Rydberg levels. This is effectively what can be observed in figure 4 where the total oscillator strength converging to a given rotational threshold is plotted, namely

$$
f^{N^{+}}(E)=\sum_{m_{\ell}} \sum_{M_{N}} \sum_{\alpha} W_{M_{N}}^{2} f_{\alpha}^{N^{+} m_{\ell}, M_{N}}(E)
$$

where $W_{M_{N}}$ is a weight for each $M_{N}$ projection in the initial state (see [11]).

Figure 4 shows the calculated spectra obtained with the approach given above for the three separate series portrayed in figure 2 . In this calculation both $l$-mixing and $n$-mixing have been taken into account. It is clear from this figure that the dominant series is indeed the $n \mathrm{p}$ converging to $N^{+}=3$. In this intense series, the $l$-mixing structure appears clearly as in the atomic Sr diamagnetic spectrum [22, 12]. This shows again that diamagnetism in $\mathrm{NO}$ can be pictured as an atomic-like effect within each rotational channel. Moreover, one can observe the gradual evolution of the competition between the Coulomb and magnetic interactions in a $1 \mathrm{~T}$ field. The $l$-mixing regime starts in the $34 \mathrm{p}$ to $40 \mathrm{p}$ energy range, then becomes fully effective in the $40 \mathrm{p}$ to $45 \mathrm{p}$ range. In this region, the $n \mathrm{p}$ levels are no longer distinguishable from the higher $\ell$ levels. However, the underlying $n \mathrm{p}$ Coulombic pattern is 


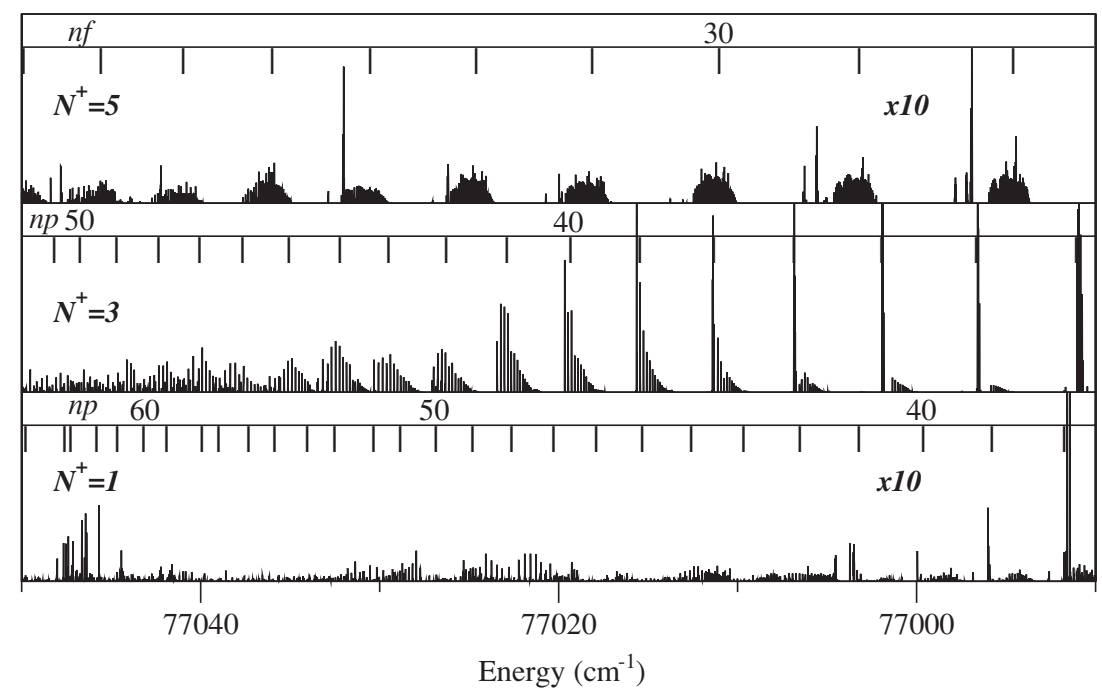

Figure 4. Contribution of the different $N^{+}$channels to the oscillator strength. Note that the weak $N^{+}=1$ and $N^{+}=5$ contributions have been magnified by a factor of 10 .

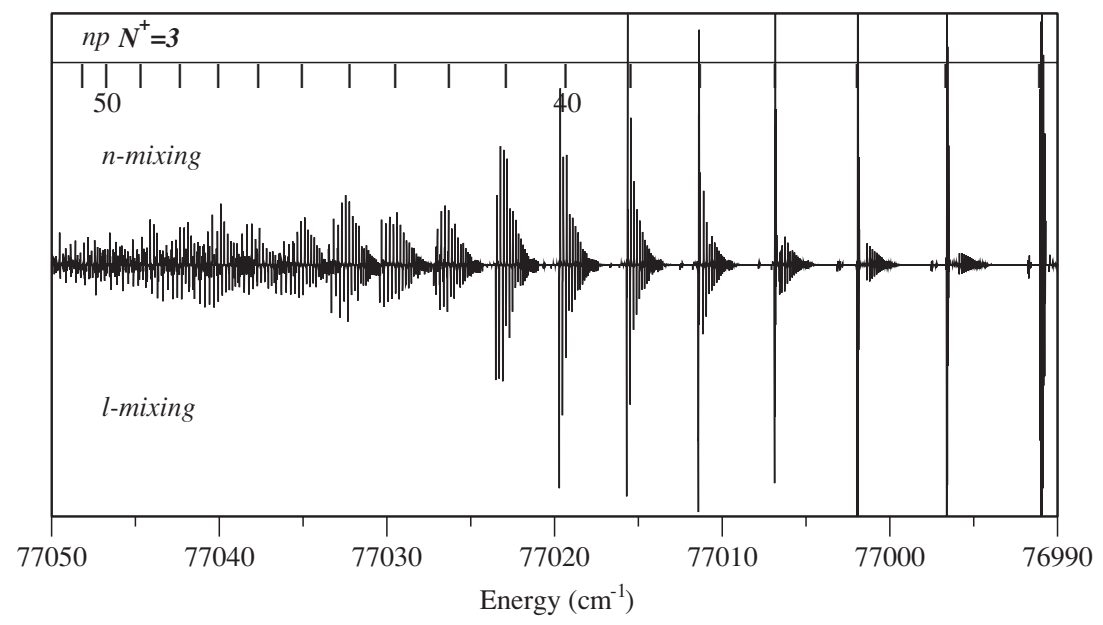

Figure 5. Comparison between $\ell$ - and $n$-mixing oscillator strengths of the $N^{+}=3$ channel contribution.

still clearly seen in this spectral range. This pattern becomes gradually scrambled when the $n$-mixing interactions take place. The effects of $n$-mixing are illustrated in figure 5 . The lower trace of figure 5 corresponds to the calculations where only $l$-mixing interactions have been included. The upper trace incorporates $n$-mixing into the calculations. Comparison between both spectra shows that $n$-mixing becomes important above $n=45$, and surprisingly, delays the scrambling of the $n \mathrm{p}$ Coulombic underlying pattern.

If we now consider the weaker $\left(n \mathrm{f}, N^{+}=5\right)$ series, the Paschen-Bach structure, observed for the lower $n$ value in our previous work, appears clearly affected by the $l$-mixing effects. These effects are already important at low $n$ values $(n=28)$ as expected for a nearly zero quantum defect $f$ series. Finally, the even weaker $N^{+}=1$ series show a complicated and diluted structure due to the strong $l$ - and $n$-mixing. In the upper half energy range, the 
validity of our perturbative treatment becomes questionable. Fortunately the contribution of the $N^{+}=1$ series to the total calculated spectrum is small and does not affect the overall aspect of the spectrum. The $N^{+}=3$ rotational channel contribution to the spectrum is so dominant that in the following section the motional Stark effect will only be discussed for the $N^{+}=3$ contribution.

\subsection{Motional Stark effect}

When experiments on Rydberg molecules in a magnetic field are carried out in a gas cell, a motional electric field is produced by the thermal motion of the molecules in the magnetic field, according to $\vec{F}=\vec{v} \times \vec{B}$, where $\vec{v}$ is the thermal velocity of the molecule. In the experiment described here (at $300 \mathrm{~K}$ ), a significant transverse motional field of the order of a few $\mathrm{V} \mathrm{cm}^{-1}$ is produced and can induce $\ell$ - and $m_{\ell}$-mixing. Such a situation is well documented in the example of the thermal Li atom by Crosswhite et al [23], later analysed by Lu and Rau using the MQDT formalism [13]. The motional Stark (MS) perturbation mixes states with $\Delta \ell= \pm 1$ and $\Delta m_{\ell}= \pm 1$. In this experiment the motional Stark effect only affects the electron motion in the plane perpendicular to the magnetic field, so that the interaction takes the following form [23]:

$$
\begin{aligned}
& \langle n(\ell-1)|r| n \ell\rangle=n\left[\left(n^{2}-(\ell-1)^{2}\right)\right]^{1 / 2} \\
& \langle n(\ell+1)|r| n \ell\rangle=n\left[\left(n^{2}-(\ell+1)^{2}\right)\right]^{1 / 2} \\
& \left\langle m_{\ell}|\cos \phi| \ell m_{\ell^{\prime}}\right\rangle=\frac{1}{2} \delta_{m_{\ell}, m_{\ell^{\prime}}=m_{\ell} \pm 1} .
\end{aligned}
$$

This motional Stark effect yields a more complicated $\ell$-mixing coupling scheme.

Figure 6 shows how this motional Stark effect enhances $\ell$-mixing and induces a dilution of the oscillator strength of a bright ( $n \mathrm{p}, m_{\ell}=0, N^{+}=3$ ) Rydberg level over several optically forbidden channels. In the absence of a motional Stark effect, as explained in the previous section, the QZE interaction (equation (12)) only dilutes the oscillator strength of the $\left(n \mathrm{p}, m_{\ell}=0\right)$ Rydberg level through all the high dark $n \ell$ levels $\left(\ell=3, \ldots, n-1, m_{\ell}=0\right)$, i.e. the levels plotted in the column labelled $m_{\ell}=0$ of figure 6 . The lift of degeneracy of these levels is due to the diagonal QZ shift (equation (9)). The weaker motional Stark effect couples the $\left(n \mathrm{p}, m_{\ell}=0\right)$ level with the two $\left(n \mathrm{~d}, m_{\ell}= \pm 1\right)$ levels. These levels are in turn coupled by the QZ interaction to all the $n \ell$ dark levels ( $\ell$ even) plotted in the column labelled $m_{\ell}=1$ and $m_{\ell}=-1$ respectively. Moreover every $n \ell$ level, plotted in these two $m_{\ell}=1$ or $m_{\ell}=-1$ columns, is coupled by the Stark interaction to any level $n, \ell \pm 1$ plotted in the two adjacent $m_{\ell}=0,2$ or $m_{\ell}=-2,0$ columns respectively. To summarize the $n \mathrm{p}$ level is in principle coupled with all the levels reported in figure 6. Consequently, all the levels corresponding to the $(-1)^{\ell+m_{\ell}}$ parity (here odd parity) are coupled by the QZ and MS interactions.

The QZ+MS interaction matrix hence requires the knowledge of the $n$ s and $n$ d Rydberg series, the $n$ s series being involved in the $(-1)^{\ell+m_{\ell}}$ even parity case. In order to simplify the evaluation of the interaction matrix, we have determined the LZ energy levels in the unitedatom approximation, namely with an average quantum defect of the low $\ell$ value $s, p, d, f$ levels $(0.23,0.7,-0.012,0.013$ respectively). Nevertheless, we keep using the oscillator strength associated with these $\mathrm{p}$ and $\mathrm{f}$ series as determined in the previous section. The $\mathrm{s}$ and $\mathrm{d}$ series converging to the $N^{+}=3$ threshold have zero intensity since they are optically forbidden from the $\mathrm{A}^{2} \Sigma$ state. The united atom approximation has been checked and justified by a great similarity between the united atom spectrum and the spectra of figures 5 and 4 .

Finally, the QZ + MS matrix structure is built up as follows. Each $\left(n, n^{\prime}\right)$ block of the QZ matrix corresponding to a single $m_{\ell}$ value must be replaced by the multiple $m_{\ell}$ structure 


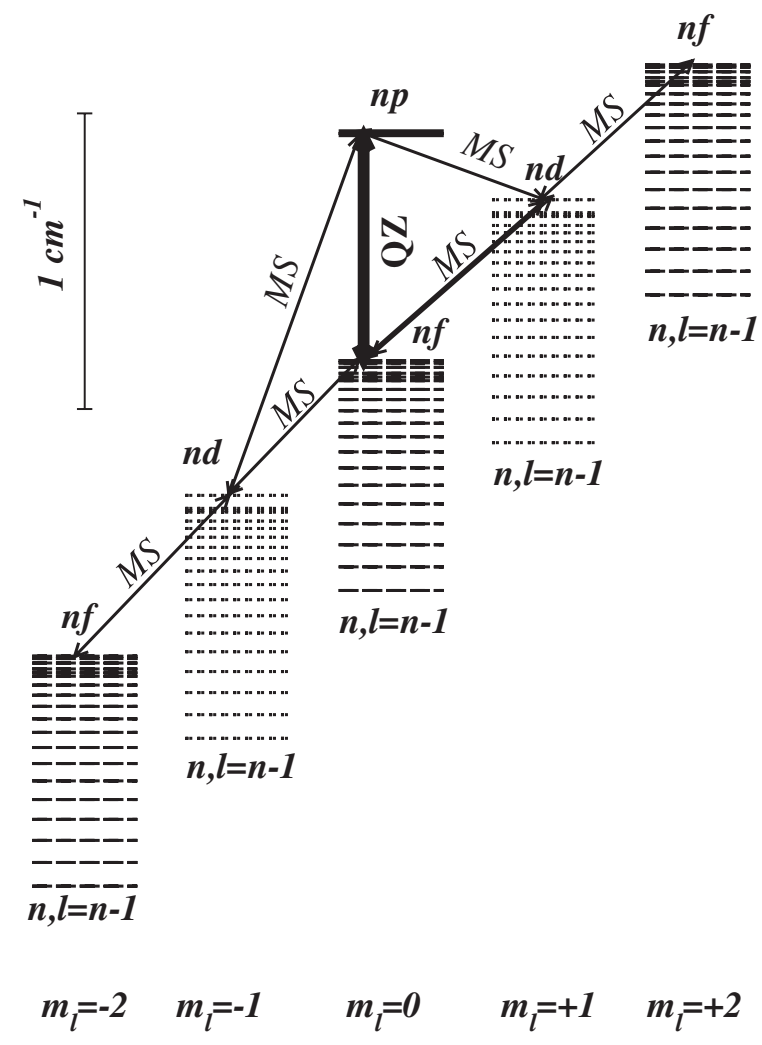

Figure 6. Illustration of the extra $\ell$-mixing induced by the motional Stark interaction in the $40 \mathrm{p}, m_{\ell}=0$ Rydberg level.

sketched in figure 6. QZ matrix elements between even $\ell$ values are determined by assuming that the radial integrals equations (10) and (12) are identical to those of the odd $\ell$ case. As expected from figure 6 and shown in figure 7, the introduction of the motional Stark effect results in a modification of the $l$-mixing intensity pattern, visible even in the lower half part of the spectral range of interest. In addition, in the upper half part of the spectrum ( $n$ above 45 ), the regularity due to the underlying $n \mathrm{p}$ structure, giving rise to intensity oscillations, is completely washed out.

To conclude, the perturbative treatment is expected to give a good description of the QZE spectra in the region of $n$ where Coulomb interaction dominates, i.e. below $n \sim 43$. This will be checked in section 5. In the high $n$ region, however, this treatment is expected to gradually break down. It is therefore of interest to look for an alternative approach that would render an interpretation of the dynamics in the high $n$ region. That is the aim of the semiclassical treatment introduced below.

\section{Semiclassical interpretation}

\subsection{Introduction}

As was pointed out in the introduction (section 1), the quadratic Zeeman effect was extensively studied in hydrogen within the framework of 'quantum chaos'. Indeed by varying the relative 


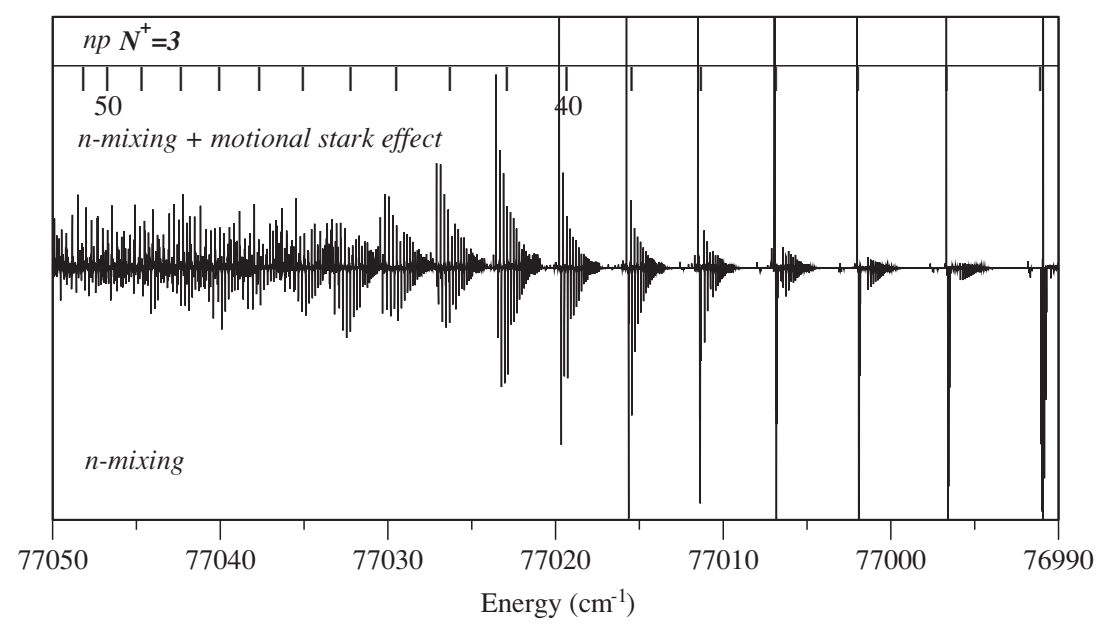

Figure 7. Comparison between $n$-mixing+motional Stark and $n$-mixing oscillator strengths of the $N^{+}=3$ channel contribution.

strengths of the Coulomb and magnetic fields, the classical motion can be either regular (near-integrable regime) or chaotic. In the former case, the spectrum of the equivalent quantum problem displays good quantum numbers, whereas in the latter case the good quantum numbers are lost and the spectrum becomes more complex (obviously this does not mean that an apparently complex spectrum necessarily belongs to a chaotic system). Semiclassical methods are generally successful in quantizing regular systems, but for chaotic systems only the large-scale fluctuations can be successfully accounted for. Still, they are extremely valuable because they are the only available tools allowing us to understand the dynamics at high energies: the fluctuations of the quantum spectra are interpreted in terms of the periodic orbits of the equivalent classical system [24]; this is best seen by taking the Fourier transform of the fluctuating part of the spectra.

In the results on NO reported in this work, we are in a transition regime from regularity to mixed dynamics. As displayed in figure 5, the molecular spectrum can be considered, in a first approximation, to be a superposition of three atomic spectra, each obtained from a core in a given rotational state. For a fixed total energy, say $E=77020 \mathrm{~cm}^{-1}$, the Rydberg electron energy is lowest, and the QZE weakest, when $N^{+}=5$. The Paschen-Back structure expected in the regular regime is still easily recognizable; indeed, the equivalent classical system is near-integrable. When $N^{+}=3$, QZE is seen to take over gradually as the energy increases: the regular structures which are visible at the lower end of the spectrum are progressively diluted as the energy of the Rydberg electron increases. Finally, when $N^{+}=1$, no regular pattern is clearly visible; the energy of the Rydberg electron at the higher end of the $N^{+}=1$ spectrum corresponds classically to a phase space with about $50 \%$ of chaotic regions (see table 1 of [25]). As detailed in section 3, the spectrum is dominated by the lines associated with the $N^{+}=3$ core state, hence in the transition regime. As was pointed out earlier, the perturbative method employed in the quantum calculations reported in this work gradually breaks down as the energy increases. Although semiclassical methods and interpretations are most useful in higher energy regimes, our aim in this section is to show how a semiclassical treatment sheds new light on the dynamics of the molecule in this transition regime, by comparing the perturbative MQDT results obtained in section 3 to a simple semiclassical calculation. 


\subsection{Theory}

We give the main ideas of closed orbit theory for Rydberg molecules in a magnetic field (which was developed in $[15,14])$ appropriate to the model used here. The dynamics underlying the photoabsorption spectra is explained in terms of closed orbits: following initial photoexcitation, the wavefunction of the excited electron propagates first in a region near the ionic core, in which the magnetic field can be neglected. Beyond this inner region, the wavefunction, written in the basis $\left|N^{+} m_{\ell}\right\rangle$ (cf equation (3)), is propagated (for each $\left|N^{+} m_{\ell}\right\rangle$ ) semiclassically along classical trajectories. Some trajectories return to the inner region, and the semiclassical wavefunction carried by those trajectories is matched to an exact wavefunction in the inner region given by a standard (field-free) MQDT expansion such as equation (2); but here, the coefficients $Z$ are obtained in the stationary phase approximation and encapsulate the classical trajectories information (the classical amplitude, action and Maslov index). Hence only the orbits closed at the origin built with the different core states in the uncoupled basis contribute to the excited wavefunction. The dipole transition is then obtained as in equation (6), but contrarily to equations (6) or (13b), the oscillator strength does not resolve individual levels but gives the large-scale structures of the spectra, that is the oscillating fluctuations present in the photoabsorption spectra. It is then appropriate to take the Fourier transform (FT) of the calculated spectrum: each orbit that would contribute by a sinusoidal modulation to the spectrum would appear as an isolated peak in the Fourier transform. Note that as in the quantum treatment, each value of $M_{N}$ (a conserved symmetry throughout) requires an independent calculation. Note also that the $\ell$ mixture of the initial A state was taken into account as explained in section 2.1, so that initial photoexcitation gives rise to outgoing $\mathrm{p}$ and f waves.

However the modulations are sinusoidal provided the classical orbits are the same within the investigated spectral range. This has been realized in theoretical and experimental studies of hydrogen and non-hydrogenic Rydberg atoms by exploiting a scaling property of the system $[26,27]$ : the underlying classical dynamics is kept constant all the way through the spectral range by keeping the quantity $E B^{-2 / 3}$ constant. This scaling property does not hold for molecules because the rotational constant does not scale with $B$, and although approximate scaling techniques could be employed [15], the NO spectrum studied in this work has been obtained at constant magnetic field. The upshot is that classical phase space is different at each energy $E$ of the spectrum, meaning that to obtain an adequate semiclassical spectrum would require classical calculations on a very tight energy grid. Moreover, since generic orbits only exist in given energy intervals and are different at each energy, a closed trajectory does not contribute by a clean sinusoidal modulation (but at best by a chirped signal) and does not show up in the FT spectrum as a peak. Although an individual orbit can be extracted from the experimental spectrum, as was done in [25], a semiclassical calculation of the unscaled spectrum is generally a heavy task.

\subsection{Results}

4.3.1. Two primitive orbits approximation. We now undertake semiclassical calculations for $M_{N}=-1$ in the energy interval 77008 to $77048 \mathrm{~cm}^{-1}$ using a simple approximation and compare them to the perturbative treatment given in section 2. We employ an approximation that is only valid at low-scaled energies in the near integrable regime and for short times: we only include the primitive orbits parallel and perpendicular to the field and their first five repetitions. These are the two fundamental orbits closed at the origin; most of the other closed orbits appear as bifurcations of the parallel and perpendicular orbits or of their repetitions when 


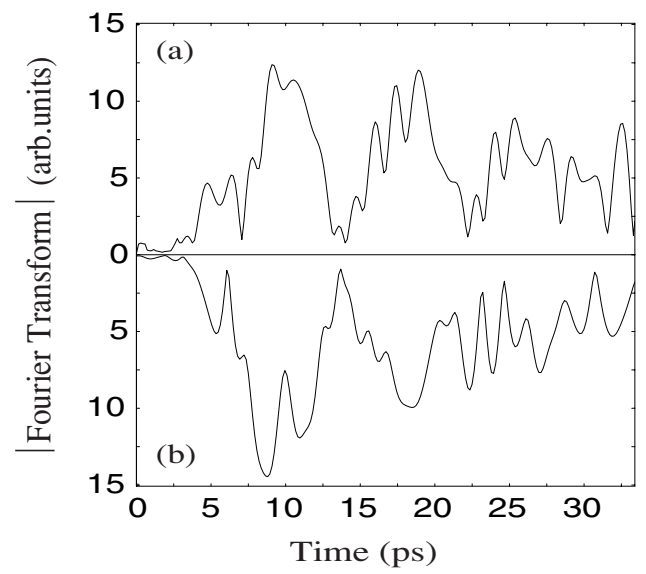

Figure 8. (a) Fourier transform of the oscillatory part of the spectrum of NO (photoexcited from the A state, $N=3, M_{N}=-1$, in a magnetic field $B=0.93 \mathrm{~T}$ ) obtained by perturbative MQDT calculations, in the energy interval 77008 to $77048 \mathrm{~cm}^{-1}$. (b) Fourier transform of the spectrum obtained semiclassically within our 'two primitive orbits approximation' (see text) in the same energy interval and $M_{N}=-1$.

the energy increases. From a computational point of view the advantage is that these orbits exist throughout the three energy intervals under scrutiny (that is for the Rydberg electron associated with $N^{+}=1,3$ and 5) and contrary to all the other closed orbits their shape is not modified when the energy is varied. The validity of this approximation is reasonable for $N^{+}=5$ (the Rydberg electron is then deeply in the near-integrable regime) but not suitable for the $N^{+}=1$ series. However, we have seen that the spectrum is dominated by the $N^{+}=3$ series, that lies in the transition regime where the two-orbits approximation starts to break down. The validity of the approximation will be tested by comparing the semiclassical results to the quantum perturbative treatment.

4.3.2. Calculations. The classical calculations involve the action, amplitude and Maslov indices obtained at different energies. Note that the action of the $n$th repetition of an orbit is simply $n$ times the action of the primitive orbit, but the amplitude and Maslov indices of repetitions do not follow trivially from the primitive orbits quantities. The action, amplitude and Maslov indices for the 12 orbits considered here are then interpolated as a function of the energy; the interpolation is unproblematic for the action, which behaves smoothly as a function of the energy. But the amplitudes as well as the Maslov indices are not well behaved; for example the Maslov index for an orbit jumps from one integer value to another at some given energy. The interpolated functions are then employed in the formulae giving the fluctuations in the oscillator strength $[15,28]$. A Fourier transform is finally taken to yield the recurrence spectrum.

The Fourier transform of the spectrum obtained quantum mechanically must be taken after subtracting the background. This is a trivial task in atomic calculations, where the background is obtained by fitting the density averaged cumulative spectral intensity to a quadratic polynomial. In the molecular case, especially in the transition regime, the Zeeman clusters at the lower end of the energy interval spoil this procedure: they induce jumps in the cumulative intensity, and the fluctuations appear embedded in large oscillations. We therefore perform a mean average smoothing over these large oscillations to extract the fluctuations; details and shortcomings of this procedure will be given elsewhere [28]. The resulting Fourier transform for short times is shown in figure $8(a)$, whereas $8(\mathrm{~b})$ shows the semiclassical results. 


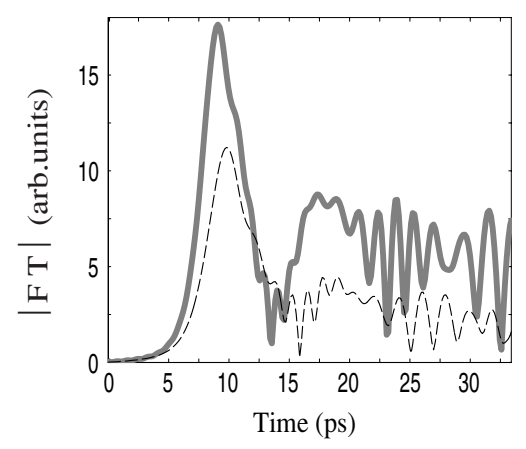

Figure 9. Main contributions to the spectrum: the grey line shows the semiclassical calculations in which only the perpendicular orbits built with the core in the rotational state $N^{+}=3$ and excited from the s $\sigma$ part of the A state have been included. The dashed line gives twice the contribution of the sole orbits parallel to the field built with the core in rotational state $N^{+}=3$ and excited from the s $\sigma$ part of the A state.

4.3.3. Discussion. It can be seen that the global structure in the semiclassical recurrence spectrum is in reasonable agreement with the quantum results, although discrepancies clearly appear when zooming in on individual peaks. As expected from figure 2 , by far the main contribution consists of orbits associated with the core in the state $N^{+}=3$. Alternatively, we can single out the contribution of a single orbit in a given energy range or of a family of orbits. We have plotted in figure 9 the most important contributions to the semiclassical recurrence spectrum: the grey line gives the contribution of the orbits perpendicular to the field (the primitive orbit and the first five repetitions) with the core in the rotational state $N^{+}=3$ and initial photoexcitation to $\ell=1$. It captures indeed most of the features seen in the total spectrum in figure $8(\mathrm{~b})$. We insist again on the fact that the peaks in the recurrence spectrum are not correlated with individual orbits at a given energy but arise from the superposition of the different orbits. For example the pattern visible in figures 8 and 9 in the interval 20 to 30 ps apparently composed of several peaks is actually mostly due to the interference of the second and third repetitions of the perpendicular orbit associated with the $N^{+}=3$ core (plotted individually, the second and third repetitions appear essentially as two very wide features centered resp. at about 20 and $30 \mathrm{ps).} \mathrm{The} \mathrm{dashed} \mathrm{line} \mathrm{shows} \mathrm{twice} \mathrm{the} \mathrm{contribution}$ of the orbits parallel to the field in the same configuration.

To sum up, the semiclassical interpretation explains the large-scale structures of the photoabsorption spectrum in terms of the contribution of closed orbits built on different core states. To increase the quantitative agreement, the classical quantities must be calculated on an tighter energy grid, more orbits must be included, and higher order effects such as combination orbits $[15,25]$ must be taken into account. However, we have seen that the simple "two orbits approximation' employed here gives a reasonable qualitative agreement with the quantum calculations. Direct comparison with the experimental spectrum is also possible, since most of the unwanted effects present in the experimental spectrum either do not affect the large-scale structures of the spectrum or can be taken into account in the semiclassical treatment. Here the main limitation concerns the fact that the experimental spectrum contains all the $M_{N}$ values ( $M_{N}=-3$ to 3 ), each subspectrum having a different weight; the procedure to extract the fluctuating part in such spectra is therefore less precise [28], and the interpretation is obscured by the fact that the overall spectrum is itself a superposition of individual spectra with a well defined $M_{N}$. This is why we have only shown here calculated spectra for $M_{N}=-1$ (which is the main subspectrum [16]). 


\section{Comparison with experiment}

\subsection{Predissociation effects}

The intensity calculations of section 3 do not take into account the well-known predissociation affecting the $n \mathrm{p}$ series of NO [29, 30]. This dynamical process is responsible for both line broadening, line shift and ionization signal decrease in REMPI experiments. This predissociation has been taken into account by introducing line broadening and shift in the $n \mathrm{p}$ series before introducing the diamagnetic interaction [31]. An average shift of $0.2 \mathrm{~cm}^{-1}$ was taken in order to fit to the experimental low $n \mathrm{p}$ line positions. The line broadening was accounted for by adding an imaginary part $\Gamma_{n}$ to the real diagonal $n \mathrm{p}$ level energies in the QZ interaction matrix. The magnitude of the effective imaginary part $\Gamma$ has been taken such that $\Gamma_{n}=0.025 \mathrm{~cm}^{-1}$ for the $n=35$ member of the $n \mathrm{p}$ Rydberg series $[29,30]$. Then a $n^{3}$ scaling law in Rydberg series was assumed for the evolution of the width with the principal quantum number, i.e. $\Gamma_{n}=\Gamma / n^{3}$. An important effect arising from the magnetic interaction is that the $n \mathrm{p}$ levels are stabilized through their interaction with the high $n \ell$ states. The predissociation process is thus gradually quenched by the QZ interaction ( $l$-mixing). Above $n=40$ when the $\ell$-mixing is fully effective, the predissociation can be considered as negligible. We anticipate that this quenching of the predissociation process gives rise to the unusual increase of the ionization signal when approaching the ionization threshold as observed in the bottom panel of figure 10 (see also figure 1 of [8]).

\subsection{Saturation effects}

In addition, as mentioned in the experimental section, partial saturation effects were observed in the intensities. The following saturation scheme, usually used to describe population saturation in the laser excitation of two-level atoms, has been adapted to account for saturation intensity effects in the bright $n \mathrm{p}$ series. Each line of the theoretical spectra has a dimensionless intensity factor (see, e.g., [32]):

$$
I_{\alpha, \text { sat }}(E)=\frac{\epsilon_{\mathrm{sat}}^{2} D_{\alpha}^{2}}{\epsilon_{\mathrm{sat}}^{2} D_{\alpha}^{2}+\left(\Gamma_{\alpha} / 2\right)^{2}+\left(E-E_{\alpha}\right)^{2}}
$$

where $\epsilon_{\text {sat }}$ is the laser electric field amplitude. This $\epsilon_{\text {sat }}$ value has been chosen in such a way that the intensity of the $n \mathrm{p}, N^{+}=3$ Rydberg series appears comparable to that of the $n \mathrm{f}, N^{+}=5$ series. Finally the saturated theoretical spectra is convoluted by the $0.07 \mathrm{~cm}^{-1}$ experimental resolution. The resulting spectra are presented and compared to the experimental one in figure 10 .

\subsection{Discussion}

Considering the relative theoretical contributions in the three different $N^{+}$channels reported above in figure 4 the experimental spectra presented in the bottom panel of figure 10 is rather surprising. One can observe that the experimental spectra correspond quite well to the underlying Coulomb pattern reported in figure 2. This pattern is recalled in this panel to help the reader follow all the members of the $n \mathrm{f}$ and $n \mathrm{p}$ Rydberg series. Our saturation scheme (equation (16)) efficiently discriminates intense and broad predissociated $n \mathrm{p}$ lines from the weak and narrow $n f$ lines.

Figure 10 helps to show the limit of our perturbative treatment: our approach appears valid when the $l$-mixing dominates, i.e. in the 76990 to $77025 \mathrm{~cm}^{-1}$ energy range. The three different approximations shown in figure 10 lead to quantitatively the same spectra. This 


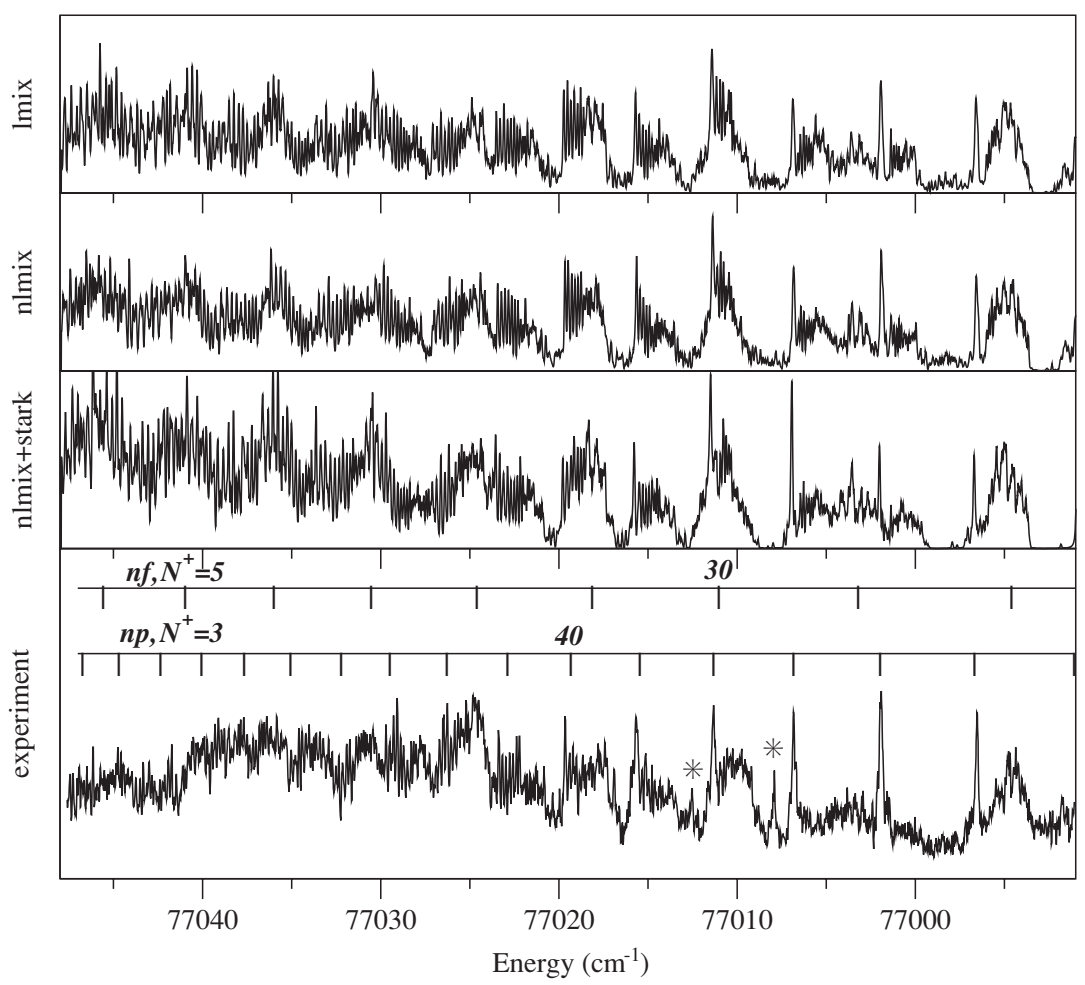

Figure 10. In the three upper panels are reported the theoretical perturbative spectra corresponding to the three rotational channel contributions calculated in three different approximations: top, l-mixing; central, $n$-mixing; and bottom, $n$-mixing + Stark motional in the united-atom approximation (see text for detail), limited to the two dominant rotational channel contributions $N^{+}=3,5$. The negligible contribution of the $N^{+}=1$ channel contribution is not included due to the excessive size of the ZQ+MS interaction matrix. In the lower panel the experimental spectrum is reported. The Coulombic pattern, corrected by the diagonal QZE shift, is recalled for the $(n \mathrm{p}$, $m_{\ell}=0, N^{+}=3$ ) and ( $n f, m_{\ell}=0, N^{+}=5$ ) Rydberg series.

incidentally shows the relatively weak influence of the motional Stark effect in this experiment. On the other hand, when the $l$-mixing is fully effective and $n$-mixing is rising, the agreement becomes less quantitative. However, the underlying Coulomb pattern is still visible in the experimental spectra in this energy range. We reach the limits of our perturbative treatment around $n \geqslant 43$.

Moreover, when the density of states becomes very high as is the case in this energy range, any interaction however weak may change the intensity pattern. In particular the weak partial wave $\mathrm{p}$ and $\mathrm{f}$ molecular mixing (breaking of spherical symmetry by the molecular potential) could give rise, via accidental near degeneracy, to extra coupling between $\left(n \mathrm{p}, N^{+}=3\right.$ ) and ( $n \mathrm{f}, N^{+}=5$ or $\left.N^{+}=1\right)$. In principle such a coupling could be taken into account in the LZE-MQDT treatment [33] as long as the QZ interaction is negligible. However, in the high $n$ regime, this molecular $\mathrm{p}$ and $\mathrm{f}$ mixing is a much smaller interaction than the $\mathrm{QZ}$ one. Perturbation theory then requires that this very weak interaction cannot be included before accounting for the strong QZ one. This would imply interaction matrices including the three rotational channels and lead to huge matrices difficult to diagonalize. The two lines indicated by asterisks in the bottom panel should be the early manifestation of such weak couplings. Our 
calculations indeed determined several levels appearing at these particular energies, but their associated intensities are far too weak. We expect such coincidences to become more frequent in the higher part of the spectrum as the energy increases. In this range, these interactions may not only have an impact on the intensity pattern but also a more pronounced effect on level positions. According to equation (8), the QZ diagonal shift of any ( $n \mathrm{p}, N^{+}=3$ ) level, weakly interacting with a quasi-degenerate $\left(n \mathrm{f}, N^{+}=1\right)\left(\operatorname{resp} .\left(n \mathrm{f}, N^{+}=5\right)\right)$, will increase (resp. decrease) [13].

\section{Conclusion}

In this work we have shown that the increasing complexity observed in the experimental spectrum does not yet mean that a chaotic regime has been reached. The moderate magnitude of the magnetic field, $B=1 \mathrm{~T}$, used in this experiment, allows us to observe a gradual distortion of the underlying Coulomb pattern. The good agreement between theory and experiment in the first part of the spectrum validates a posteriori this perturbative approach. However, slight intensity disagreements appear in the higher part of the spectra, due to the very high density of states, since any tiny coupling may substantially modify the intensity pattern.

In this work, we have particularly studied the influence of the motional Stark effect, the first weak interaction that must be considered when in the experimental setup the magnetic field is not parallel to the molecular beam. Our study shows that the MS effect is present in the experiment but does not influence that much the general intensity pattern governed essentially by the competing QZ and Coulomb interactions. In the higher part of the spectrum where this treatment starts to break down gradually, the present approach is still helpful in understanding the origin of the main features observed in the spectra. Our perturbative treatment clearly shows that this complexity arises from the interplay between essentially two weakly interacting Rydberg series converging towards two different rotational thresholds.

The present perturbative approach cannot be generalized straightforwardly to most other Rydberg molecules. The agreement obtained in this work is partly due to the relatively weak anisotropy of the NO molecular field. Our perturbative treatment should have been more difficult to implement in the $\mathrm{H}_{2}$ molecule where rotational interchannel couplings are stronger than in the NO case. For the low $\ell$ values, those that are associated with the levels carrying the oscillator strength, the QZE interaction matrix should no longer be evaluated only within a single dressed $N^{+} m_{\ell}$ rotational channel, as assumed in this work, by simply inspecting the largest channel contribution to the total wavefunction (see figure 3). Several $m_{\ell}$ values and $N^{+}$channels will necessarily need to be included in order to give a correct description of the QZ interaction matrix. In other words, the molecular effects described in this paper are rather weak, and generally much stronger intensity effects must be expected in more anisotropic molecules.

In summary, we want to stress the major difficulties in getting a reliable intensity description in the case of high density of states, which are coupled by three competing interactions whose relative magnitudes vary strongly with the excitation energy. To achieve, in the molecular case, the same exceptionally good agreement with experiment that was reached by calculations for Rydberg atoms in a magnetic field would require calculations larger by several orders of magnitude. This is in principle feasible following the $R$-matrix method, implemented in $\mathrm{H}_{2}$ [6]. However, an $R$-matrix treatment accounting for both the QZE and MS interactions is certainly not an easy task. Moreover it is already known from the atomic case that the quantum calculations at high Rydberg electron energies, while reproducing the intensity pattern observed experimentally, become black boxes, as they give no clue to interpret the underlying dynamics. This is why we have also presented a semiclassical treatment which 
can explain the underlying physics in the upper part of the spectrum, closer to the ionization threshold.

\section{References}

[1] Delande D, Bommier A and Gay J C 1991 Phys. Rev. Lett. 66141

[2] Iu C H, Welch G R, Kash M M, Kleppner D, Delande D and Gay J C 1991 Phys. Rev. Lett. 66145

[3] Watanabe S and Komine H 1991 Phys. Rev. Lett. 673227

[4] Watanabe S, Hosoda Y and Komine H 1992 Phys. Rev. A 462693

[5] O’Mahony P F and Mota-Furtado F 1991 Phys. Rev. Lett. 672283

[6] Monteiro T S and Taylor K T 1990 J. Phys. B: At. Mol. Opt. Phys. 23427

[7] Takezawa K and Abe H 1999 J. Chem. Phys. 1109492

[8] Takezawa K and Abe H 1999 J. Chem. Phys. 11011682

[9] Fano U 1970 Phys. Rev. A 2353

[10] Monteiro T S and Taylor K T 1989 J. Phys. B: At. Mol. Opt. Phys. 22 L191

[11] Raoult M, Guizard S and Gauyacq D 1991 J. Chem. Phys. 958853

[12] O’Mahony P F and Taylor K T 1986 Phys. Rev. Lett. 572931

[13] Lu K T and Rau A R P 1983 Phys. Rev. A 282623

[14] Matzkin A and Monteiro T S 2001 Phys. Rev. Lett. 87143002

[15] Matzkin A, Dando P A and Monteiro T S 2002 Phys. Rev. A 66013410

[16] Guizard S, Shafizadeh N, Horani M and Gauyacq D 1991 J. Chem. Phys. 947046

[17] Gauyacq D, Raoult M and Shafizadeh N 2002 The Role of Rydberg States in Spectroscopy and Photochemistry ed C Sandorfy (Dordrecht: Kluwer)

[18] Cheung W Y, Chupka W A, Colson S D, Gauyacq D, Avouris P and Wynned J J 1983 J. Chem. Phys. 783625

[19] Kaufman K, Nader C and Jungen M 1985 Chem. Phys. 95385

[20] Oertel G K and Shomo L P 1968 Astrophys. J. Suppl. 16175

[21] Garstand R H 1977 Rep. Prog. Phys. 40105

[22] Lu K T, Tomkins F S and Garton W R S 1978 Proc. R. Soc. A 362421

[23] Crosswhite H, Fano U, Lu K T and Rau A R P 1979 Phys. Rev. Lett. 42963

[24] Gutzwiller M C 1990 Chaos in Classical and Quantum Mechanics (New York: Springer)

[25] Matzkin A, Raoult M and Gauyacq D 2003 Phys. Rev. A 68061401

[26] Wintgen D 1987 Phys. Rev. Lett. 581589

[27] Holle A, Main J, Wiebusch G, Rottke H and Welge K H 1988 Phys. Rev. Lett. 61161

[28] Lombardi M, Matzkin A and Raoult M in preparation

[29] Anezaki Y, Ebata T, Mikami N and Ito M 1984 Chem. Phys. 89103

[30] Raoult M 1987 J. Chem. Phys. 874736

[31] Fano U 1961 Phys. Rev. 1241866

[32] Grynberg G, Aspect A and Fabre C 1997 Introduction aux Lasers et à l'Optique Quantique (Paris: Ellipses)

[33] Greene C H and Jungen Ch 1985 Adv. At. Mol. Phys. 2151 